\title{
MODULARITY AND PARALLEL KINEMATICS: AN ORIGINAL DESIGN METHODOLOGY APPLIED TO HIGH PRECISION
}

\author{
MURIELLE RICHARD, PROF. REYMOND CLAVEL \\ Laboratoire de Systèmes Robotiques (LSRO), Ecole Polytechnique Fédérale de Lausanne \\ (EPFL), Station 9, CH-1015 Lausanne, Switzerland
}

\begin{abstract}
This paper introduces a new methodology to design modular industrial ultra-high precision robots; it aims at significantly reducing both the complexity of their design and their development time. This modular concept can be considered as a robotic Lego®, where a finite number of building bricks is used to quickly design the robot and to easily change its mobility. The core of the concept is the thorough conceptual solution catalogue, which is independent from any mechanical design. This paper will first present the methodology and the techniques to establish this solution catalogue. Then, its application to high precision will include the formulation of hypotheses and a practical example of a 5-degree of freedom ultra-high precision robot design.
\end{abstract}

\section{Motivation}

The current trend in numerous industrial domains is to miniaturize products, mainly microelectronic, optic and biomedical devices; the design of industrial robots capable of performing micromanipulation and micro-assembly tasks with a sub-micrometric precision is thus becoming a crucial need [1]. At the present time, the R\&D process to design and build such ultra-high precision machines is still costly. Therefore, design methodologies have been developed in order to reduce the time-to-market, for example in [2]. However, if the industrial specifications of the robot are modified, requiring for instance a change in its mobility, the whole design process has to be restarted from the beginning.

This paper thus presents an original concept of modular kinematics which allows to rapidly design a parallel robot and to modify only a small part of the kinematics to change its mobility. This approach can be compared to a robotic Lego ${ }^{\circledR}$, where a finite number of conceptual bricks can be chosen and assembled within a small amount of time to create the desired structure, thanks to a conceptual solution catalogue [3]. In parallel with the conceptual aspects, the mechanical design of the building bricks is investigated, making use of flexure hinges: this type of joints is indeed compulsory to meet the submicrometric precision requirements [4]. Consequently, the robots resulting from 
this methodology achieve similar high performances as more traditionally designed machines, with a significantly shortened development time.

This paper will first present the modular design methodology, especially the steps of the orderly process generating the conceptual solution catalogue. Then, its application to high precision robots will be detailed: hypotheses linked to the mechanical flexure-based design of the bricks will be introduced, and an ultrahigh precision 5-DOF (Degrees of Freedom) robot design will illustrate the practical use of the methodology.

\section{Modular design methodology}

\subsection{Theoretical bases and terminology}

This methodology consists in designing a parallel robot composed of one to three kinematic chains, which are orthogonally arranged. This robot is symbolized by a cube: the chains are disposed along its faces, while the endeffector is located on one of its corners. The design of this robot makes use of a finite number of conceptual building bricks, which present two states: active and passive. Each kinematical chain of the modular robot consists in the serial arrangement of an active brick with a passive one.

As their name indicates, the role of the active bricks is to actuate from one to three DOF; the motions which are not actuated are blocked. As for the passive bricks, their function is to link the output of the active bricks to the end-effector of the robot, thus achieving a transmission of the motion. Their DOF are either passive, i.e. free to move but not actuated, or blocked. A generic notation uniformly represents the building bricks by symbolizing their two main features (see figure 1). First, it indicates their mobility: $\mathrm{T}$ represents a translation and $\mathrm{R}$ stands for a rotation. Uppercase letters symbolize actuated motions, whereas lowercase letters stand for passive ones. Then, the notation also specifies the directions of the DOF in relation to the face of the cube on which the brick is located: $\perp$ indicates that the motion is along an axis which is orthogonal to the plane of the face, whereas a motion along one of the directions of that plane is represented by II. Likewise, the subscripts 1 and 2 are used to explicitly express the directions of several DOF along both possible axes of that plane.

The methodology transforms the desired mobility of the robot into an exhaustive list of all possible combinations of active and passive bricks fulfilling it, with the help of a conceptual solution catalogue. Then, the most suitable kinematics for the considered problem is selected depending on the robot specifications, as well as on mechanical design considerations. 
The generation of the exhaustive conceptual solution catalogue comprises two main steps: first, the thorough list of the active bricks arrangements actuating the desired mobility is established; then, for each of them, all combinations of passive bricks performing the transmission of the motions without overconstraints are itemized. The next paragraphs will detail both of these steps.

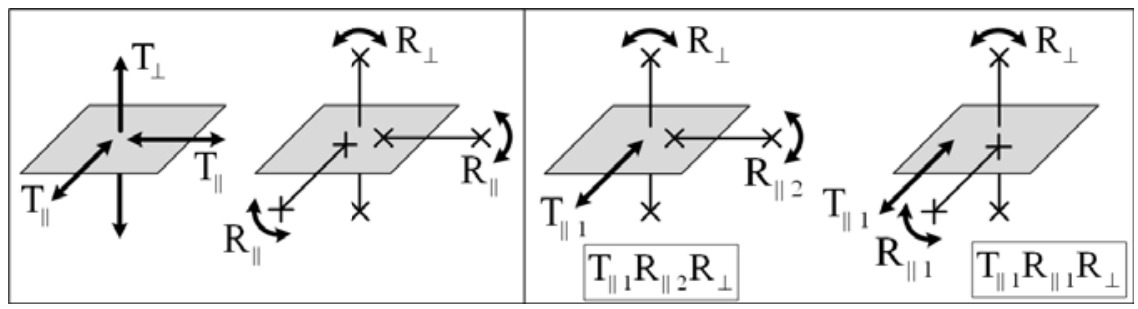

Figure 1. Illustration of the building bricks notation system (left) and examples of correspondence between brick mobility and notation (right)

\subsection{First step: active bricks arrangements}

The generation of the catalogue first consists in listing all possibilities of arranging one to three active bricks on the cube, so that they actuate the motions which the robot has to perform. It is to note that the permutations of the same bricks along the cube faces are listed as one solution. An intuitive approach first consists in establishing the different families of solutions which perform the required motions. For instance, a kinematics performing a planar joint motion (two translations and a rotation along three different axes) present the following ones:

1. 1 active brick, actuating 2 translations and 1 rotation

2. 2 active bricks, the first actuating 2 translations, the second 1 rotation

3. 2 active bricks: the first actuating 1 translation and 1 rotation, the second 1 translation

4. 3 active bricks, two of them performing 1 translation, the third 1 rotation

Then, for each of these families, all bricks actuating the required motions are combined: nonetheless, the consistency of these arrangements with the concept definition must be verified. Indeed, the second family of the previous example admits four bricks: $T_{\|} T_{\perp}$ and $T_{\| 1} T_{\| 2}$ to actuate two translations, $R_{\|}$and $R_{\perp}$ for the rotation. A maximum of four combinations is thus possible, among which only three are compatible with the concept: the $T_{\| 1} T_{\| 2}$ and $R_{\perp}$ solution is impossible, as it would require both bricks to be located on the same face of the cube (see figure 2). 


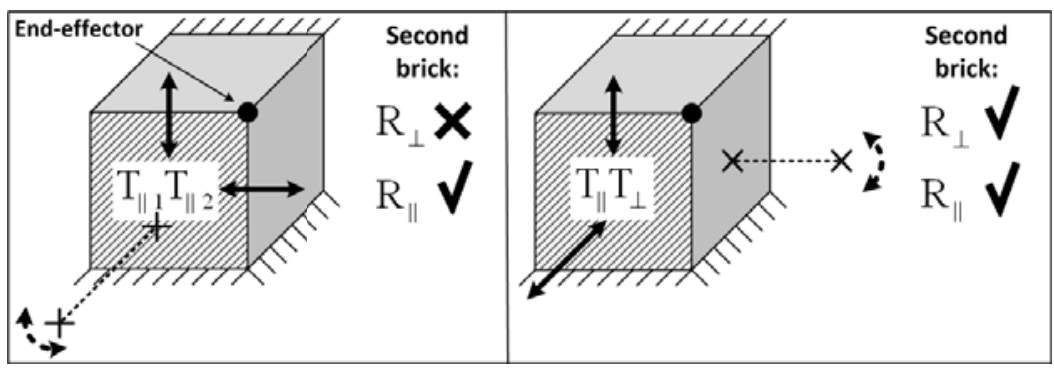

Figure 2. Second solution family of the plane joint mobility: the first brick, performing both translations, is arbitrarily placed on one face of the cube; the second brick has to actuate the remaining rotation, symbolized by a dashed line.

This first approach has allowed to establish the conceptual solution catalogue; moreover, a second method has been developed to automatically generate the solutions, making use of combinatory algorithms to produce the arrangements: first, a list of all active brick combinations is established. Then, three dismissing algorithms are applied: the first suppresses the solutions actuating more than once the same DOF, whereas the second deletes the combinations which are not compatible with the concept, for instance the ones where two bricks should be located on the same face of the cube. Last, all permutations of the same arrangement are dismissed. These methods have been implemented on Matlab, which has allowed to prove the thoroughness of the solution catalogue established with the first approach.

\subsection{Second step: passive bricks arrangements}

Once the exhaustive active bricks arrangements are listed, the second step consists in determining which passive bricks should be added to each of them to obtain the required robot DOF. The mobility of each passive brick is established as following: first, to perform the transmission of the actuated motions, the passive brick has to block the DOF which are actuated by the serially arranged active brick. Then, the motions which are actuated by the other active bricks of the robot (if any) must be free to move. Last, the remaining motions must avoid overconstraints, i.e. must ensure that the position of each blocked DOF is imposed by only one passive brick. In other words, the movements must be blocked on one passive brick and remain passive on the others. Figure 3 illustrates the determination of the passive bricks for a specific solution of the planar joint example (see 2.2). 


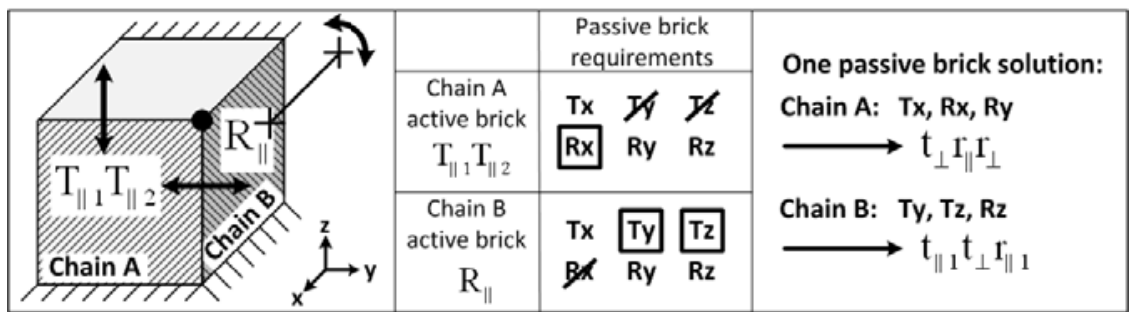

Figure 3. Example of the determination of the passive bricks solutions: planar joint mobility, active bricks: $T_{\| 1} T_{\| 2}$ and $R_{\|}$. The crossed motions represent the DOF which are actuated by the corresponding active brick, whereas the framed DOF stand for the ones which are actuated by the other active brick.

The total number of solutions of the exhaustive conceptual solution catalogue exceeds 3000, which makes it hardly applicable to a real design context. Consequently, simplifying hypotheses must be formulated: these are based on the robot requirements, especially on the mechanical design of the building bricks. The next paragraphs will illustrate possible assumptions for the specific case of ultra-high precision robots.

\section{Application to ultra-high precision}

Ultra-high precision robots refer to machines which are capable of performing micromanipulation and micro-assembly tasks with a resolution of less than 10 nanometers. To achieve this requirement, their mechanical design must be without friction, backlash and wear: the use of flexure hinges is thus mandatory. These joints are based on the elasticity of the material itself and can be monolithically machined by Wire Electro-Discharge Machining. The mechanical design of the bricks and some machining considerations thus allow the formulation of simplifying hypotheses.

\subsection{Hypotheses for high precision}

For high precision applications, the active bricks are assumed to be planar flexure-based mechanisms, which are motorized by linear actuators only, the rotations being obtained by the differential motions of the actuators of the same active brick. Consequently, only the conceptual active bricks which can shrewdly be designed with flexure joints are considered.

Likewise, the passive bricks are assumed to be designed as planar or axisymmetric structures. As the shrewdness of their integration in a high 
precision robot highly depends on the combination of the active and passive bricks within the kinematic chain, all conceptual passive bricks are allowed for.

These hypotheses first transform the complex 3D robot design problem, which results in scarcely modular structures, into several 2D ones, which are simpler and well-mastered. Then, the aforementioned dismissal of some active bricks allows to establish a simplified conceptual solution catalogue for high precision, thus dividing by two the number of kinematical possibilities. More details on the mechanical design of the building bricks can be found in [5].

\section{Application of the methodology to a 5-DOF robot}

The choice of the 5-DOF mobility is motivated by the typical industrial applications of high-precision robots, for instance micromanipulation tasks. These often require three translations (Tx, Ty, Tz) and two rotations (Rx, Ry), the rotation around the axis of the robot tool being blocked. This kinematics is complex to design and manufacture, which explains the scarcity of industrial prototypes. The hypotheses which are formulated for this problem are the following:

1. Active bricks: all bricks presenting the same mobility with different orientations relatively to the face of the cube share the same design; the physical plane of the brick does thus not necessarily match the face of the cube.

2. The robot presents a cubic arrangement of its kinematic chains.

3. Passive bricks: the mechanical design of each brick must belong to a plane which is parallel to the one of the corresponding active brick.

4. The number of different building bricks must be minimized.

5. The robot must be easily transformable into a 4-DOF or a 6-DOF one.

\subsection{Choice of the kinematical solution}

The use of the simplified conceptual solution catalogue results in only one cubic active brick arrangement for each family, as the first hypothesis causes solutions which are conceptually different to result in the same mechanical design. Then, one passive brick solution is selected for each active brick arrangement: its choice is based on the machinability of the selected mechanical designs of the passive bricks and on the third hypothesis. Figure 4 illustrates the

four remaining kinematics: solution 4 is preferred as it minimizes the number of building bricks (hypothesis 4). Figure 5 illustrates the principle of the robot design, as well as a double-scaled mock-up which has been built with assembled blades. 


\begin{tabular}{|c|c|c|c|c|c|}
\hline \multirow{5}{*}{ X-chain } & & X-chain & Y-chain & Z-chain & \\
\hline & \multirow{2}{*}{ Solution 1} & $\mathrm{~T}_{\| 1} \mathrm{~T}_{\| 2} \mathrm{R}_{\perp}$ & $\mathrm{T}_{\|} \mathrm{R}_{\perp}$ & $\varnothing$ & \multirow{2}{*}{$\begin{array}{c}4 \text { different } \\
\text { bricks }\end{array}$} \\
\hline & & $\mathrm{t}_{\perp} \mathrm{r}_{\|}$ & $\mathrm{t}_{\| 1} \mathrm{t}_{\perp} \mathrm{r}_{\| 1} \mathrm{r}_{\| 2}$ & $\varnothing$ & \\
\hline & \multirow{2}{*}{ Solution 2} & $\mathrm{~T}_{\| 1} \mathrm{~T}_{\| 2} \mathrm{R}_{\perp}$ & $\mathrm{R}_{\perp}$ & $\mathrm{T}_{\|}$ & \multirow{2}{*}{$\begin{array}{l}6 \text { different } \\
\text { bricks }\end{array}$} \\
\hline & & $\mathrm{t}_{\perp} \mathrm{r}_{\| 1} \mathbf{r}_{\| 2}$ & $\mathrm{t}_{\| 1} \mathrm{t}_{\| 2} \mathrm{t}_{\perp} \mathrm{r}_{\| 1} \mathrm{r}_{\| 2}$ & $t_{\| 1} t_{\perp} r_{\| 1} r_{\| 2}$ & \\
\hline & \multirow{2}{*}{ Solution 3} & $\mathrm{~T}_{\|} \mathrm{R}_{\perp}$ & $\mathrm{R}_{\perp}$ & $\mathrm{T}_{\| 1} \mathrm{~T}_{\| 2}$ & \multirow{2}{*}{$\begin{array}{l}6 \text { different } \\
\text { bricks }\end{array}$} \\
\hline & & $\mathrm{t}_{\| 1} \mathrm{t}_{\perp} \mathrm{r}_{\| 1} \mathrm{r}_{\| 2}$ & $t_{\| 1} t_{\| 2} t_{\perp} r_{\| 1} r_{\| 2}$ & $\mathrm{t}_{\perp} \mathbf{r}_{\| 1} \mathbf{r}_{\| 2}$ & \\
\hline & \multirow{2}{*}{ Solution 4} & $\mathrm{~T}_{\|} \mathrm{R}_{\perp}$ & $\mathrm{T}_{\|} \mathrm{R}_{\perp}$ & $\mathrm{T}_{\|}$ & \multirow{2}{*}{$\begin{array}{l}3 \text { different } \\
\text { bricks }\end{array}$} \\
\hline & & $\mathrm{t}_{\| 1} \mathrm{t}_{\perp} \mathrm{r}_{\| 1} \mathrm{r}_{\| 2}$ & $\mathrm{t}_{\| 1} \mathrm{t}_{\perp} \mathrm{r}_{\| 1} \mathrm{r}_{\| 2}$ & $\mathrm{t}_{\| 1} \mathrm{t}_{\perp} \mathrm{r}_{\| 1} \mathrm{r}_{\| 2}$ & \\
\hline
\end{tabular}

Figure 4. The four remaining kinematics of the 5-DOF robot

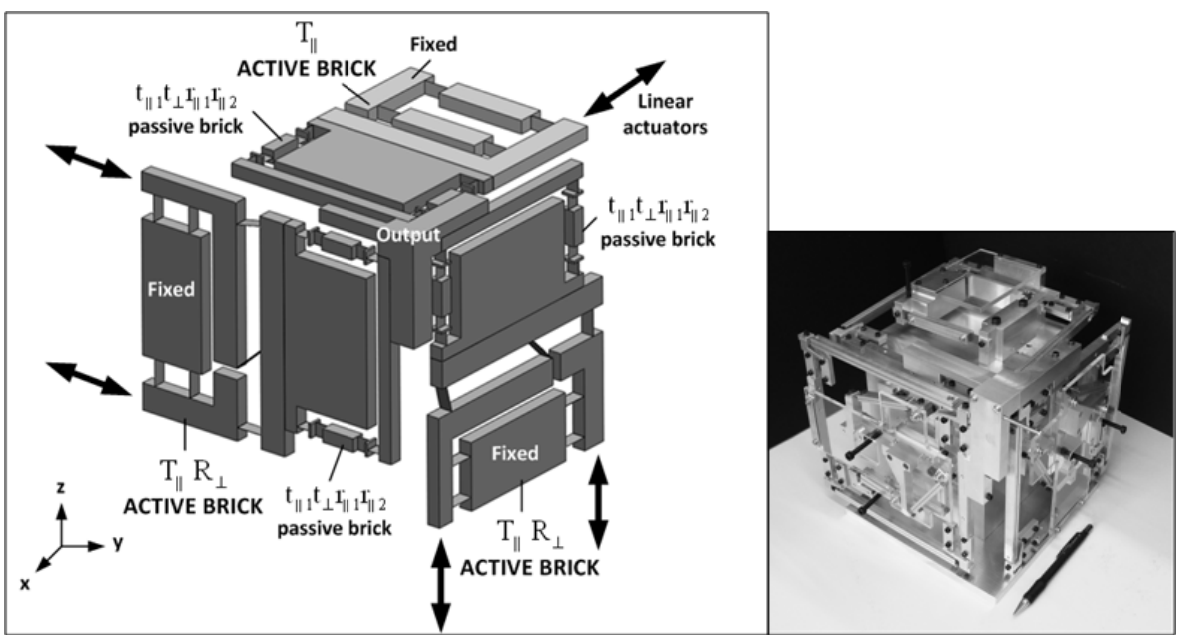

Figure 5. Design principle (left) and double-scaled mock-up (right) of the 5-DOF Legolas 5 robot

The fifth hypothesis is also fulfilled as the mobility of this kinematics can straightforwardly be modified: the substitution of the $T_{\|}$brick by a $T_{\|} R_{\perp}$ indeed results in a 6-DOF robot, whereas the opposite operation creates a 4-DOF one. This application of the methodology has thus generated a new family of ultrahigh precision robots, named Legolas: with only the four building bricks of the 5-DOF robot (thus named Legolas 5) and a simple passive one, all 19 possible DOF arrangements can be performed. This family notably includes the orthogonal version of the Delta kinematics [6, 7]. 


\section{Conclusion}

This paper has introduced an original methodology to design high precision modular robots. This robotic Lego®, based on a finite number of building bricks and on a conceptual solution catalogue, allows to rapidly develop a robot and to easily modify its mobility. This paper has detailed the methods to generate the exhaustive conceptual catalogue, as well as possible simplifying hypotheses based on high precision specific requirements. These have been applied to a practical example consisting in the development of a 5-DOF robot, which has highlighted the creation of a new family of ultra-high precision parallel machines. The design of an industrial prototype of the Legolas 5 is currently in progress; its characterization will allow to prove that the performances of the robots resulting from this new methodology are at least similar to the ones of more classically designed kinematics.

\section{References}

1. D. Tolfree, 'Commercialising Nanotechnology, concepts - products markets', International Journal of Nanomanufacturing, vol. 1, $\mathrm{n}^{\circ} 1, \mathrm{pp}$. 117-133, 2006.

2. P. Helmer, 'Conception systématique de structures cinématiques orthogonales pour la microrobotique', Ph.D. thesis $n^{\circ} 3365$, Swiss Federal Institute of Technology Lausanne (EPFL), 2006.

3. M. Richard, R. Clavel, 'A new concept of modular kinematics to design ultra-high precision flexure-based robots', Proceedings for the joint conference of the $41^{\text {st }}$ International Symposium of Robotics and the $6^{\text {th }}$ German Conference on Robotics, Munich, pp. 940-947, 2010.

4. S. Henein, 'Conception des guidages flexibles', Presses Polytechniques et Universitaires Romandes, Collection Meta, Lausanne, 2001.

5. M. Richard, R. Clavel, 'Concept of modular flexure-based mechanisms for ultra-high precision robot design', Mechanical Sciences, vol. 2, 99107, 2011.

6. J.-P. Bacher, S. Bottinelli, J.-M. Breguet, R. Clavel, 'Delta3: a New Ultrahigh Precision Micro-Robot: Design and Control of a Flexure Mechanism', Journal Européen des Systèmes Automatisés, vol. 36, nº/2002, ed. Lavoisier: France, pp. 1263-1275, 2002.

7. S. Bottinelli et al., 'Movement transmission unit and movement transmission apparatus employing the same', US Patent US006453566B1, September 24th, 2002. 